Revista de la Escuela de Ciencias de la Educación, año 13, nRo. 12, vol. 2, julio a diciembre de 2017. Páginas 13-33. ISSN 1851-6297. ISSN 2362-3349 (EN LINEA). LA SENSIBILIDAD POR LAS VIOLENCIAS EN EL ESPACIO ESCOLAR DESDE EL DIScurso mediático. Virginia Saez y Verónica Soledad Silva

\title{
LA SENSIBILIDAD POR LAS VIOLENCIAS EN EL ESPACIO ESCOLAR DESDE EL DISCURSO MEDIÁTICO
}

\author{
Por Virginia Saez \\ Universidad de Buenos Aires, Argentina. \\ saezvirginia@hotmail.com \\ Verónica Soledad Silva* \\ Universidad de Buenos Aires, Argentina. \\ verosilva13@yahoo.com.ar
}

Recibido: 19/09/2016 Aceptado: 28/12/2016

\begin{abstract}
Resumen
El artículo presenta los hallazgos de una investigación socioeducativa sobre los discursos mediáticos. El objetivo es analizar la mediatización del caso conocido como la Masacre de Carmen de Patagones sucedido en el año 2004 en Argentina. Se basa en el análisis cualitativo de una muestra de ciento cincuenta y dos notas de los diarios de la ciudad de La Plata, en el período 20042006. La fuente de datos fue secundaria y se realizó una muestra finalística, seleccionando las notas que aportaran información de interés en relación al
\end{abstract}

Doctora en Educación por la Universidad de Buenos Aires, Magister en Educación: Pedagogías Críticas y Problemáticas Socioeducativas, Licenciada en Ciencias de la Educación y Profesora en Enseñanza media y superior en Ciencias de la Educación por la Universidad de Buenos Aires (UBA). Becaria postdoctoral del CONICET con sede en el Instituto de Investigaciones en Ciencias de la Educación de la Universidad de Buenos Aires (UBA). Miembro del Programa de Investigación sobre Transformaciones Sociales, Subjetividad y Procesos Educativos dirigido por la Dra. Carina V. Kaplan.

* Licenciada en Psicología, Universidad Nacional de Córdoba. Becaria doctoral del CONICET, Instituto de Estudios Sociales y Humanos (IESyH). Universidad Nacional de Misiones (UNaM) - Facultad de Humanidades y Ciencias Sociales. Doctoranda del Doctorado en Educación, Facultad de Humanidades y Ciencias de la Educación de la Universidad Nacional de La Plata. Miembro del Programa Transformaciones sociales, Subjetividad y Procesos educativos dirigido por la Dra. Carina Kaplan. 
objetivo estipulado. El método utilizado es el análisis socioeducativo del discurso. La presente investigación amplía la base empírica con la que analizar y problematizar cómo es representada la escuela, sus actores y los episodios de violencia en los medios de comunicación. Los resultados evidencian que desde la prensa los episodios de violencia en las escuelas son construidos desde una perspectiva individualizante, a través de las voces autorizadas por especialistas y apelando a un discurso emotivo, que obstaculizan análisis más complejos.

Palabras Clave: Violencia - Escuela - Medios de Comunicación - Jóvenes

\section{Abstract}

The article presents the findings of an investigation on the speeches rehabilitative media. The objective is to analyze the media coverage of the case known as the massacre of Carmen de Patagones happened in the year 2004 in Argentina. It is based on the qualitative analysis of a sample of one hundred and fifty-two notes of the diaries of the city of La Plata, in the period 20042006. The data source was secondary and conducted a sample finalística, by selecting the notes that provide information of interest in relation to the aim stipulated. The method used is the analysis of socio-educational discourse. The present investigation extends the empirical basis with which to think and discuss the difficulty of the representation of the school and its actors in the media. The results show how to construct the episodes of violence in schools from a perspective individualizante, through the authoritative voices by specialists, and appealing to an emotional speech that prevents more sophisticated analysis.

Key words: Violence - School - Mass Media - Young

\section{Introducción}

El periodismo cuenta con una posición privilegiada en la producción de discursos sociales dado que dispone de los medios más potentes para hacerlos circular e imponerlos (Bourdieu, 2002). Las noticias periodísticas, en tanto construcciones de la realidad (Verón, 1981), producen y reproducen principios de visión y división del mundo social. Los modos por los cuales la prensa construye el vinculo entre violencia y escuela como parte de una agenda mediática generan efectos que reproducen discursos e imágenes sobre la escuela y los sujetos que la habitan (Bourdieu y Wacquant, 2005). Dada esta impronta, el presente artículo tiene por objetivo analizar la mediatización del caso conocido como la Masacre de Carmen de Patagones sucedido en el año 2004 en Argentina. 
Revista de la Escuela de Ciencias de la Educación, año 13, nRo. 12, vol. 2, JUlio a diciembre de 2017. Páginas 13-33. ISSN 1851-6297. ISSN 2362-3349 (EN LINEA). LA SENSIBILIDAD POR LAS VIOLENCIAS EN EL ESPACIO ESCOLAR DESDE EL DIS-

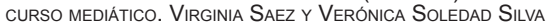

Dicho acontecimiento representó un hito en la cobertura mediática sobre el fenómeno de las violencias en la escuela por varias razones: fue el único caso que tuvo una continuidad de tres años en la cobertura periodística; a partir de este suceso empezaron a mediatizarse diversos hechos de violencias en la escuela en todo el territorio argentino, en muchos casos refiriendo a un fenómeno de imitación; cuando la prensa nacional nomina episodios de violencia sucedidos a escala internacional alude a este caso como referencia. Consideramos que la Masacre de Carmen de Patagones produjo un cambio en las cartografías de la violencia en el espacio escolar delimitando un antes y un después en la cobertura mediática sobre las violencias en la escuela.

Ahora bien, ¿por qué analizar los discursos mediáticos? Los medios de comunicación ocupan posiciones estratégicas en la lucha de los significados sociales, en tanto producen, reproducen, legitiman y deslegitiman discursos y representaciones sobre la realidad y los sujetos. A partir de los noventa, autores como Delgado (1998), Alba (2002), Saintout (2009) y Vasilachis de Gialdino (2004) observan que los medios construyen el estereotipo de un sujeto peligroso mediante procesos de selección noticiosa y estrategias discursivas que operan como mecanismos de control social. Para los autores, este interés mediático reafirma un tipo de consenso social basado en alentar el pánico moral contra la inseguridad, encarnada en la figura desviada de la juventud (Núñez, 1999; Brener, 2009).

La fuente utilizada en esta investigación es la prensa gráfica, en tanto el propósito es analizar cómo los medios de comunicación reproducen y crean sentidos sobre la escuela y los sujetos que la habitan en un público que va más allá de las instituciones formadoras. En continuidad con la Pedagogía Crítica (Giroux, 1997) sostenemos que la educación es una práctica política social y cultural, y por ello toma especial relevancia las tensiones que se producen entre ese afuera escolar (los medios de comunicación, las voces autorizadas que allí circulan) y lo propio de las instituciones educativas. En este sentido, nos preguntamos acerca de cómo los medios de comunicación participan en la construcción de la identidad social de la escuela y sus actores y en la representación/sensibilización de cierta configuración emotiva sobre los fenómenos de las violencias en la escuela.

\section{Aspectos metodológicos}

Desde una perspectiva socioeducativa se considera que las violencias en la escuela no son un correlato mecánico de la violencia social, aunque es allí donde se originan y cobran su sentido más hondo. Ciertas mediaciones intervienen en la escuela para que esta posibilite algo distinto que los comportamientos brutales que suscitan las sociedades capitalistas salvajes (Kaplan, 2009). En este marco, el presente trabajo busca caracterizar y analizar la mediatización del caso conocido como la Masacre de Carmen de Patagones, en 
Revista de la Escuela de Ciencias de la Educación, año 13, nRo. 12, vol. 2, Julio a diciembre de 2017. PÁginas 13-33. ISSN 1851-6297. ISSN 2362-3349 (EN LíNEA). LA SENSIBILIDAD POR LAS VIOLENCIAS EN EL ESPACIO ESCOLAR DESDE EL DISCURSo mediático. ViRginia Saez y Verónica Soledad Silva

tanto constituye un hito en la mediatización de los episodios violentos en el espacio escolar.

El abordaje metodológico es cualitativo, y el diseño de investigación asume un carácter exploratorio. A partir de un muestreo intencional se seleccionaron las prácticas discursivas que aportaran información de interés en relación al objetivo estipulado. El estudio es diacrónico y analiza cómo se ha producido la visibilización de la Masacre de Carmen de Patagones a lo largo del período comprendido entre 2004 y 2006.

El año de inicio del relevamiento fue seleccionado porque en 2004 acontece el incidente a analizar y finaliza en el 2006 porque es allí donde aparece la última nota sobre el episodio, en las fuentes relevadas.

El corpus se conforma por ciento cincuenta y dos notas de los principales diarios de la ciudad de La Plata: sesenta y cuatro del diario El Día, sesenta y nueve del diario Hoy y diecinueve del diario Extra.

Es necesario destacar que se eligen los medios gráficos dado que son el medio de referencia dominante y que marcan tendencia informativa para el resto de los medios de comunicación (Bonilla Vélez y Tamayo Gómez, 2007) y porque interesa la materialidad de los diarios dada la potencialidad del texto escrito para observar las distintas formas de nominación del fenómeno. Se seleccionaron los diarios de la ciudad de La Plata porque es la capital de la provincia de Buenos Aires y es considerada su principal centro político, administrativo y educativo. Estas características la hacen significativa del presente estudio, por ser un espacio social a partir del cual se toman decisiones que afectan a todo el país. Asimismo, el equipo de investigación en el que participamos desarrolla el trabajo de campo en escuelas secundarias de esa zona.

El análisis de este episodio es de relevancia, dado que en una investigación más amplia (Saez, 2016) se evidencia cómo la aparición del mismo constituye el pico más alto de la cobertura mediática de la prensa platense en el periodo 1993-2011 (1).

Gráfico 1: Cantidad de notas del caso Carmen de Patagones de la prensa platense 1993-2011

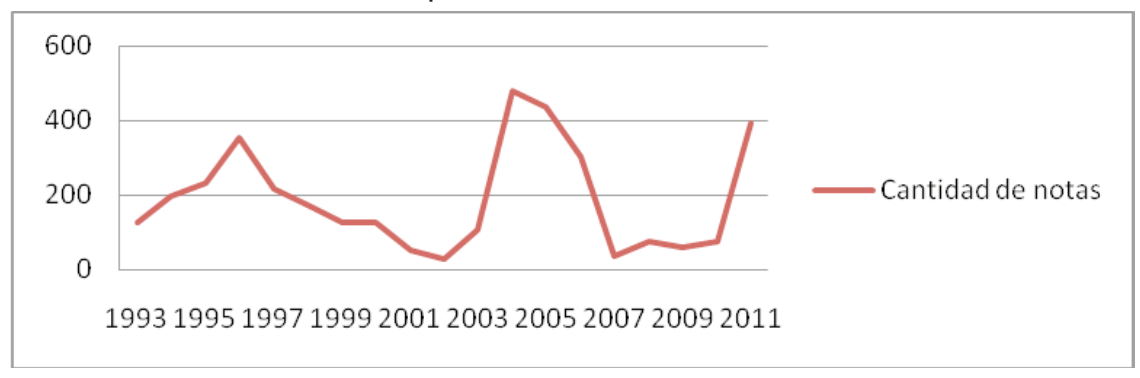

Fuente: Tesis doctoral de Saez (2016). 
La "Masacre de Carmen de Patagones" marcó un hito y se transformó, para la prensa de La Plata, en el principal punto de referencia de la presentación de la violencia en el ámbito escolar. Así también, fue el único caso en que los medios seleccionados hicieron un seguimiento durante tres años, 2004, 2005 y 2006. En la siguiente tabla se especifica la cantidad de notas publicadas.

\begin{tabular}{|c|c|}
\hline Año & Cantidad de notas \\
\hline 2004 & 94 \\
\hline 2005 & 45 \\
\hline 2006 & 13 \\
\hline
\end{tabular}

En los medios relevados se distribuyeron de la siguiente manera:

Gráfico 2: Distribución de notas del caso Carmen de Patagones en la prensa platense 1993-2011.

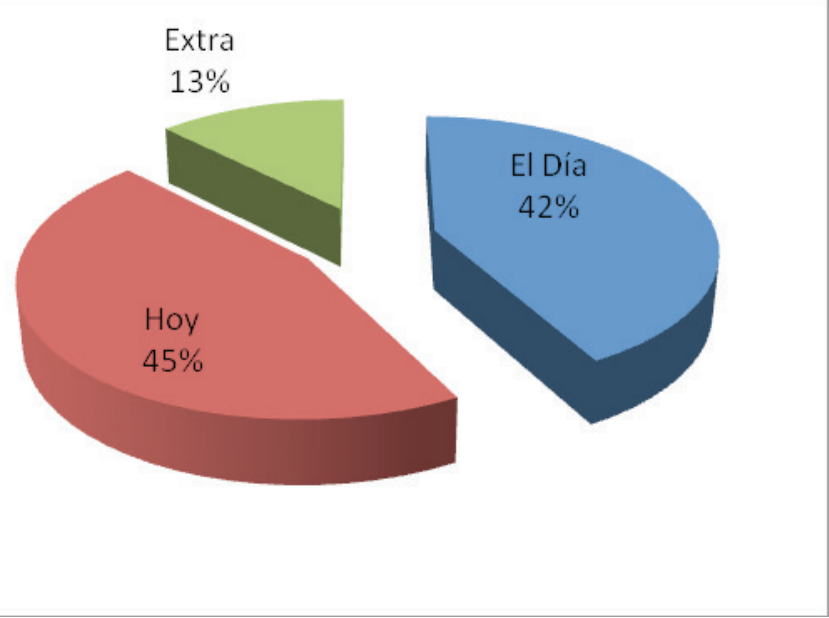

Fuente: Elaboración propia a partir de los datos de la presente investigación.

Se expondrá a continuación una breve referencia de cada uno de los periódicos incluidos en la muestra, que permiten comprender la diferencia en la cantidad de notas del corpus.

El diario El Día fue fundado en la ciudad de La Plata el 2 de marzo de 1884. Se convirtió en el primer órgano de prensa de la naciente capital de la provincia de Buenos Aires, que apenas tenía quince meses de vida cuando el 
diario empezó a editarse. A lo largo del tiempo, El Día se consolidó como diario líder de la capital bonaerense y llegó a ser una referencia fundamental en la prensa escrita del país.

Por su parte, el diario Hoy nació el 10 de diciembre de 1993. Según el matutino, cuenta con una tirada de sesenta y cinco mil ejemplares diarios, lo que implica doscientos cincuenta mil lectores potenciales.

$Y$ en tercer término, es de particular interés explicitar que el diario Extra se elabora e imprime en La Plata y se distribuye al interior del país como anexo de otros diarios locales. Por lo tanto no tiene como principales lectores a los ciudadanos platenses. Dicho medio nace en el 2003, como un diario-complemento para diarios locales o regionales, con el objetivo de incrementar la calidad de la información, internacional y nacional, incluyendo la deportiva, y disminuir sensiblemente los costos.

El tratamiento de los datos se realizó en el marco del análisis socioeducativo del discurso (Martín Criado, 2014). Esta metodología es de relevancia para

...dilucidar el juego de tensiones y ambivalencias en que se mueven prácticas y discursos (...) y ver las estrategias simbólicas para legitimar o deslegitimar a los distintos sujetos y sus prácticas -de ahí el énfasis en situar todo discurso en un espacio de discursos y todo enunciado en la estrategia general de presentación de sí (Martín Criado, 2014, p. 133).

La línea de análisis que se sigue postula una interrelación entre las noticias y la práctica social que los produce, por eso referiremos a práctica discursiva. Desde esta perspectiva el uso de ciertos actos de nombramiento y clasificación en las notas estará determinado por las convenciones socialmente aceptadas para el discurso en el que se inserta. A su vez, los límites de ese discurso estarán relacionados con las condiciones de reproducción/transformación que posibilitan las estructuras sociales existentes.

\section{Las violencias en las escuelas desde el campo representacional de los medios de comunicación}

La problemática de las violencias (2) en la escuela adquirió en los últimos años en Argentina un gran protagonismo en la opinión pública: en los medios de comunicación, en el ámbito académico y en las políticas públicas.

Específicamente, analizar el lugar de los medios de comunicación en las configuraciones sociales contemporáneas, adquiere significación para reflexionar acerca del lugar que ocupan en la construcción de la sensibilidad por las violencias en el espacio escolar. A través de sus prácticas discursivas los medios explican, ordenan y califican, dichos acontecimientos. Variadas investigaciones ponen de manifiesto que éstos tienden a visibilizar las temáticas sobre la escuela y sus actores desde sus falencias (Iglesias, 2015; Saez, 2015) y de forma difusa y contradictoria (Núñez, 1999). 
Revista de la Escuela de Ciencias de la Educación, año 13, nRo. 12, vol. 2, JUlio a diciembre de 2017. Páginas 13-33. ISSN 1851-6297. ISSN 2362-3349 (EN LINEA). LA SENSIBILIDAD POR LAS VIOLENCIAS EN EL ESPACIO ESCOLAR DESDE EL DIScurso mediático. Virginia Saez y Verónica Soledad Silva

Los medios crean y recrean una forma de sensibilidad específica frente a la problemática de la violencia (Kaplan, 2011), promoviendo representaciones que incluyen a los jóvenes en hechos de violencia ocurridos en o asociados por implicación al escenario escolar, imponiendo con evidencia creciente en la opinión pública la sensación de una escuela atravesada por la violencia (Míguez y Noel, 2006; Unicef y FLACSO, 2011). Cabe destacar que la violencia es un valor - noticia determinante en las agendas periodísticas latinoamericanas (Consejo Nacional de Televisión de Chile, 1998 y 2002; Herrera 1998; López y Cerda, 2001; Comfer, 2005).

Junto con Kaplan (2011) sostenemos que las percepciones que la sociedad tiene sobre la violencia están vinculadas a una sensibilidad epocal.

La violencia hoy es a la vez un problema público y una inquietud personal de nuestra época. Y no ha sido así infaliblemente (...) la sensibilidad por la violencia es cultural y se vincula en su génesis a los procesos civilizatorios de pacificación en los inicios de las sociedades estatales y a los procesos de urbanización (Kaplan, 2011, p.71).

Cuando hablamos de proceso civilizatorio, nos referimos a la conceptualización desarrollada por Norbert Elias (2009) (3) quien desde una investigación de larga duración (4) busca reconstruir las conexiones entre las estructuras psicológicas individuales y las estructuras sociales. La tesis central del autor es que a partir del aumento de las cadenas de interdependencia y la progresiva centralización de las formas de organización social, más específicamente, con la constitución de los Estados modernos al centralizarse el monopolio de la violencia física, se produce la pacificación de las relaciones humanas al interior de las sociedades (Kaplan y Silva, 2016). Como señala Míguez: "La idea de proceso civilizatorio, supone una dinámica que ha atravesado a casi la totalidad de la cultura occidental durante varios siglos, moderando los niveles de agresión entre los integrantes de la sociedad" (2009, p.148). Esto no quiere decir que la violencia desapareció de los vínculos interpersonales, sino que hubo un proceso de desplazamiento de expresiones de violencia física hacia otras formas "más sutiles" de violencia, pero no por ello menos efectivas.

A este respecto decimos que como resultado del proceso civilizatorio, algunas prácticas que en otros momentos nos eran indiferentes o concebidas como naturales, ahora nos resultan aberrantes y dignas de condena social. Una perspectiva sociohistórica y procesual (Elias, 2009) resulta fértil para la indagación del fenómeno de las violencias en el espacio escolar, entendiendo a su vez, que las prácticas discursivas de los medios hunden sus significaciones en la cultura.

Sostenemos que hay una interrelación entre las percepciones acerca de las violencias y los umbrales de sensibilidad, en esta interrelación consideramos que los medios ocupan un rol central 
(...) no siempre nos afectó la violencia del mismo modo ni con la misma intensidad que hoy, y digamos que tampoco estamos en condiciones de anticipar qué percibiremos más adelante. La sensibilidad por la violencia es, por tanto, biográfica e histórica y lo es en el entramado de configuraciones particulares donde expande su sentido más hondo (Kaplan, 2011, p.77).

Los sentidos sobre la violencia se encuentran situados social, cultural e históricamente, vale decir, su definición habrá de variar en el tiempo, en el espacio y de acuerdo con diversos contextos sociales (Garriga Zucal y Noel, 2010). En otras palabras, la violencia solo es definible en los términos en que cada colectividad la concibe (Isla y Míguez, 2003). Por tanto, los comportamientos individuales requieren ser abordados en una matriz social, histórica y procesual (Elias, 2009).

Una de las vías de análisis sobre el fenómeno de las violencias en la escuela desde el campo de los medios de comunicación, es la consideración de que las percepciones sobre las mismas se vinculan a la menor tolerancia de ciertas prácticas que quizás antes eran vivenciadas como naturales y que ahora quebrantan lo que suponemos que los vínculos interpersonales deberían ser.

A este respecto consideramos que los medios de comunicación al producir bienes simbólicos suministran un insumo significativo a los individuos para la construcción de sus imaginarios (Ford, 1999). Por tanto, la información periodística y los imaginarios sociales se estimulan y contaminan mutuamente (Baczko, 1991). Estos, a través del poder de construcción de realidad (Verón, 1987), proveen a los sujetos del conocimiento del mundo social, al que no pueden acceder de manera directa (Martini, 2004). Los medios de comunicación, por tanto, abonan a la construcción de una sociedad atravesada por múltiples violencias, síntoma de una incivilización que irrumpe en el normal desarrollo de una sociedad pensada como a conflictiva (Garriga Zucal y Noel, 2010).

A partir de este poder simbólico (Bourdieu, 1988 y 2001), los medios se sitúan como formadores de opinión en la sociedad. Bourdieu señala que el campo periodístico "debe su importancia en el mundo social a que detenta un monopolio de hecho sobre los instrumentos de producción y de difusión de gran escala de la información" (1998, p.17). Entendiendo el poder simbólico como el poder de nombrar y de construir/producir visiones y divisiones sociales (Bourdieu, 1999). Es el derecho sobre la producción de sentido, sobre la definición y prescripción de lo social, como realidad social.

Para el autor, los medios de comunicación pueden convertirse en una herramienta de manipulación y en un "formidable instrumento de dominación" (Bourdieu, 2005, p. 79). Los nombres de las cosas sociales son objeto de lucha simbólica; de allí que es necesario examinar el proceso de constitución y legitimación de ciertas formas de clasificación que operan en el mundo social. 
Revista de la Escuela de Ciencias de la Educación, año 13, nRo. 12, vol. 2, julio a diciembre de 2017. Páginas 13-33. ISSN 1851-6297. ISSN 2362-3349 (EN LINEA). LA SENSIBILIDAD POR LAS VIOLENCIAS EN EL ESPACIO ESCOLAR DESDE EL DIScurso mediático. Virginia Saez y Verónica Soledad Silva

La información periodística constituye una fuente privilegiada para el análisis de las configuraciones emotivas en determinado tiempo histórico y social.

\section{Un hito que deja huella sobre las representaciones de la escuela}

En nuestro trabajo de investigación nos encontramos con la irrupción de un acontecimiento (Foucault, 2004) en el discurso mediático: La Masacre de Carmen de Patagones. Incidente ocurrido el 28 de septiembre de 2004 en una escuela secundaria de Carmen de Patagones, al sudoeste de la Provincia de Buenos Aires, Argentina, en el cual un alumno de 15 años llevó un arma de fuego y disparó contra sus compañeros de aula, provocando tres víctimas fatales y cinco heridos. Este hecho conmocionó a todo el país y tuvo una repercusión social muy grande, no sólo por su persistencia en la prensa nacional, sino también porque a partir de dicho acontecimiento, se creó el Observatorio de Violencias en las Escuelas dependiente del Ministerio de Educación de la Nación (5). Este organismo está dedicado a la investigación, la enseñanza y la difusión de temas vinculados con la violencia en los colegios, asimismo tiene por objetivo la capacitación de profesionales del ámbito educativo y la elaboración de políticas públicas que tiendan a la prevención.

Pensar este hecho como un acontecimiento es un modo particular de aproximarnos a la problemática de las violencias en el espacio escolar. El objetivo de esta indagación no es la historia y los hechos en sí mismos sino establecernos en este acontecimiento para indagar los campos de sentido de la problemática de las violencias en las escuelas en el campo mediático.

La aparición de este acontecimiento es parte de un proceso histórico. Como sostiene Foucault (1992) "Nadie es pues responsable de una emergencia, nadie puede vanagloriarse [de producir un acontecimiento]; ésta se produce siempre en el intersticio" (p.16). Así, el objetivo de este trabajo no será subsumirlo bajo un gran patrón explicativo, sino, por el contrario, captar su singular constitución.

En este sentido, la investigación toma las notas periodísticas como expresión de un discurso social que circula más allá de los medios de comunicación. Las notas sobre el caso, en tanto conjuntos de acontecimientos discursivos, toman sentido en las condiciones de existencia que determinan la materialidad propia del enunciado (Foucault, 2004). En continuidad, se consideran como prácticas específicas en un régimen de condiciones históricas de emergencia de enunciados, inscriptos en una red heterogénea de relaciones, que se entrecruzan para unir las prácticas discursivas y la episteme de una época. La apelación a especialistas de la rama biomédica o psicológica y a funcionarios como voces autorizadas es una característica de la configuración que toma el saber en una época.

A continuación analizamos este episodio desentramando la red de discursos presente en las notas periodísticas que imprimen un alto impacto emotivo 
y cognitivo en la construcción de la identidad social de la escuela y sus actores. Consideramos que desde la lógica periodística se obtura la posibilidad de un análisis de mayor complejidad acerca de hechos de este calibre, apelando a explicaciones lineales e individualizantes.

Una particularidad de la mediatización de este episodio fue la convocatoria a distintas voces autorizadas: la de funcionarios (jueces, gobernadores, entre otros) y la de especialistas (psiquiatras, psicólogos, piscomotricistas, entre otros). Surge la pregunta acerca de qué efectos tiene la apelación de los periodistas al discurso autorizado para argumentar sus posicionamientos en el discurso mediático sobre las violencias en el espacio escolar.

Cuanto más autorizada oficialmente esté la figura encargada de emitir los juicios probabilísticos, como lo es el caso de los jueces (Hoy, 01-10-2004; El Día, 08-10-2004) y el gobernador (Hoy, 10-07-2004), es presumible que más significativa sea su capacidad de incidir sobre la imagen que se construye de los actores escolares.

A continuación se presentan algunos fragmentos interesantes que dejan huella de esto:

"La jueza pretende saber si Junior tenía acceso a material violento". (Hoy, 08-10-2004).

"ENTREVISTA AL JUEZ DE MENORES JULIO BARDI. Es alarmante que a mayor riesgo social haya menor contención familiar. El magistrado platense afirma que los menores no adquieren valores esenciales ni contención inmediata en el núcleo familiar. Explica la 'Tragedia de Patagones' por la ausencia de prevención y represión en sentido legal: 'El colegio no puede inculcar lo que la casa y el Estado no les ofrecen."'(Hoy, 01-10-2004).

"En este clima, el gobernador Felipe Solá manifestó su oposición a la adopción de medidas de seguridad extremas en los colegios. 'No podemos poner un detector de armas en las puertas de las escuelas y no podemos asumir, a partir de episodios violentos, una actitud policial', dijo Solá, inclinándose por reforzar los mecanismos para 'el seguimiento de las personalidades que puedan estar sufriendo una fuerte frustración, para ir sobre ellas rápidamente desde el punto de vista psicológico'. 'No hagamos que el dolor nos haga buscar culpables... lo que ocurrió en Carmen de Patagones es la locura de un chico', remató el Gobernador". (Hoy, 30-09-2005).

"La jueza Alicia Ramallo dio algunos detalles sobre los primeros informes psiquiátricos del autor de la Masacre de Carmen de Patagones. Dijo que 'sabía lo que hacía', pero 'no está en su sano juicio'. Quedará alojado en un neuropsiquiátrico. Se reunió con su familia. La opinión de juristas platenses". (Hoy, 01-10-2004). 
Revista de la Escuela de Ciencias de la Educación, año 13, nRo. 12, vol. 2, JUlio a diciembre de 2017. Páginas 13-33. ISSN 1851-6297. ISSN 2362-3349 (EN LINEA). LA SENSIBILIDAD POR LAS VIOLENCIAS EN EL ESPACIO ESCOLAR DESDE EL DIScurso mediático. Virginia Saez y Verónica Soledad Silva

Vemos de este modo cómo el recorte periodístico va construyendo un relato sobre el hecho apoyándose en los discursos autorizados. Éstos ejercen efectos de poder y muchas veces favorecen a legitimar el orden establecido y producir mayor eficacia simbólica a su acción (Bourdieu, 1997). En este caso se apela por momentos a cierta responsabilización de los "valores" que debe inculcar la familia o el Estado, como así también el apelativo a que la única explicación es la "locura de un chico" que "no está en su sano juicio", valores y locura se entraman en un discurso confuso y fragmentario sobre el hecho.

Si nos detenemos en los enunciados que comportan las voces de los científicos observamos lo siguiente:

“JUNIOR, SEGÚN LOS PSIQUIATRAS: 'Él sabia bien lo que hacía, pero no está en su sano juicio'. Un brote y una despersonalización pudieron haber sido las causas de la reacción violenta de Junior. 'Él deseaba algo de esto pero en el momento no fue consciente, entre comillas. Hay algo de desconocimiento de lo que él había hecho', explicó el psicomotricista Esteban Levin, y destacó que 'este chico tendría que haber sido atendido antes, ya que con un tratamiento esto se habría podido prevenir'. 'Cuando lanza los trece disparos está en absoluto descontrol y después, cuando lo enfrenta su amigo, de alguna manera reacciona porque se detiene, no le tira al compañero y entrega el arma, no se resiste', agregó el especialista". (Hoy, 01-10-2004).

"Habian advertido problemas psicológicos". (Hoy, 03-10-2004).

¿Por qué aparecen determinadas voces autorizadas para hablar sobre las violencias en el espacio escolar? Esto nos lleva a analizar qué expertos se mencionan en las coberturas mediáticas del corpus. Resulta relevante que los profesionales que aparecen son exclusivamente de la rama biomédica o psicológica. Como sostiene Bourdieu (2006), estas prácticas discursivas, que se mencionan como la voz de los científicos, son discursos con pretensión de verdad capaces de ejercer violencia simbólica imponiendo arbitrariedades culturales con un alto grado de legitimación.

Otra estrategia utilizada por los diarios fue la preeminencia de una mirada individualizante en torno a las posibles soluciones para abordar esta temática.

Se constató que las posibles soluciones que circularon en las noticias refieren al orden de lo individual, focalizando en la figura del victimario, su estructura psicológica y el posible destino del adolescente (la cárcel o el neuropsiquiatrico), su inimputabilidad ante la ley, la peligrosidad que reviste para la sociedad, entre otros.

Veamos algunas citas:

"CARMEN DE PATAGONES. CAMBIO DE ESTRATEGIA. Qué pasará con el chico que disparó. (...) No será derivado a un instituto. Estará bajo tratamiento". (Hoy, 01-10-2004). 
Revista de la Escuela de Ciencias de la Educación, año 13, nro. 12, vol. 2, julio a diciembre de 2017. Páginas 13-33. ISSN 1851-6297. ISSN 2362-3349 (EN LINEA). LA SENSIBILIDAD POR LAS VIOLENCIAS EN EL ESPACIO ESCOLAR DESDE EL DISCURSo mediático. ViRginia Saez y Verónica Soledad Silva

"El pibe que mató a tres compañeros en una escuela de Patagones fue derivado a una clínica del conurbano. Estaba alojado en el Instituto de Menores de El Dique, donde habría sido agredido por otros internos. Ayer circuló el rumor de que se había autoinfligido tres cortes con una madera, pero fuentes de minoridad lo desmintieron". (Extra, 11-06-2005).

"Trasladaron a Junior a un neuropsiquiátrico. El chico que mató a tres compañeros y baleó a otros cinco abandonó el Instituto de Menores de El Dique. Los otros internos lo habrían agredido. ¿Quedará libre?” (Hoy, 11-05-2005).

"La jueza de menores de Bahía Blanca dictó su sobreseimiento, ya que cuando mató a tres compañeritos e hirió a otros cinco tenía 15 años. Eso lo convierte en inimputable. Ahora estará tutelado por el Estado, en una causa asistencial. El viernes pasado lo trasladaron a un neuropsiquiátrico. (...) La causa penal por la tragedia en la escuela de Carmen de Patagones, donde un alumno mató a tiros a tres compañeros e hirió a otros cinco en septiembre del año pasado, quedó cerrada debido a que, por ser menor, el atacante es inimputable". (Hoy, 15-05-2005).

“ ¿Otro nombre para su vida en libertad? Junior. El caso quedó en manos de la jueza Alicia Ramallo. Lo sugirió Tomás Ponce, padre de una de las víctimas. Dijo que tras el cierre de la causa penal, el pibe que mató a tres compañeritos en Carmen de Patagones 'podrá sentarse en el banco de otro colegio' ". (El Día, 16-06-2005).

En el proceso de hacer visible el destino del victimario también aparece un discurso que apela al impacto emotivo poniendo foco en el dolor de los familiares de las víctimas y la soledad del adolescente que cometió el hecho. Observemos:

"Sepultaron los restos de los tres adolescentes asesinados. Todos marcharon por paz y justicia. Mientras tanto, Rafael está en una unidad de Prefectura. No lo visitó ni su familia”. (Hoy, 30-09-2004).

"El papá de Federico Ponce, uno de los chicos que mató Junior, se refirió al supuesto intento de suicidio del múltiple homicida. 'No vale la pena que muera, sino que viva el dolor eterno', dijo, y rogó que no sea liberado". (Hoy, 12-06-2005).

De este modo observamos que las soluciones al episodio visibilizadas en las notas abonan a los discursos individualizantes. Sin embargo, como venimos sosteniendo desde el inicio de este artículo, desde una perspectiva socioeducativa crítica sobre la temática (Kaplan, 2006, 2009, 2013), la violencia no puede ser entendida como una propiedad intrínseca de sujetos que se vuelven victimarios, sino que es siempre relacional, socialmente construida, y refiere a una multiplicidad de fenómenos y representaciones sociales. Consideramos que más allá de ciertas patologías que podrían dar lugar a conductas 
Revista de la Escuela de Ciencias de la Educación, año 13, nRo. 12, vol. 2, julio a diciembre de 2017. Páginas 13-33. ISSN 1851-6297. ISSN 2362-3349 (EN LINEA). LA SENSIBILIDAD POR LAS VIOLENCIAS EN EL ESPACIO ESCOLAR DESDE EL DIScurso mediático. Virginia Saez y Verónica Soledad Silva

violentas personales, lo cierto es que, aun en esos "casos individuales" es preciso ampliar la base interpretativa de la acción y situarla en los modos de socialización que cada sociedad privilegia y legitima.

Señalamos, que no se trata de deslindar las responsabilidades que cada uno como ciudadano tiene respecto de las consecuencias de sus actos, sino de proponer la posibilidad de interrogarnos acerca de aquellos fenómenos que exceden una explicación lineal y monocausal. Como señala Bleichmar (2008) "...nuestra sociedad es tolerante con formas de subjetivación donde el sujeto es considerado a partir de su desempeño y no de su capacidad de enlace con los demás" (p.97). En este sentido, una de las cuestiones que llamaron la atención sobre este caso, fue que Junior, era un estudiante que no causaba "problemas" en el cotidiano escolar, sus prácticas no eran disruptivas y por tanto nadie había reparado en ciertas prácticas que pudieron haber sido una señal tal vez para prevenir este hecho. Existe una red policausal o una desmultiplicación causal del acontecimiento (Foucault, 1983). La Masacre de Carmen de Patagones se instala en un campo de batalla donde las fuerzas operantes se cruzan y deslizan, se tensionan y coaccionan para darle surgimiento.

Sobre este aspecto, es dable destacar que desde la mirada mediática, la escuela aparece constantemente como un actor impotente, carente de lograr una efectiva integración de los estudiantes que asisten cotidianamente.

Otro aspecto relevante de la Masacre de Carmen de Patagones fue la presencia de un arma de fuego en la escuela como objeto amenazante. Investigaciones antecedentes sobre violencia en la escuela y medios de comunicación (Lavena, 2002, Brener, 2009) dan cuenta de que la imagen dominante de la "violencia escolar" se concentra en las agresiones entre estudiantes secundarios, mediadas por la utilización de armas. Por su parte, desde las percepciones de los estudiantes, relevadas en investigaciones anteriores por nuestro equipo de investigación, se muestra cómo la portación de armas es la situación que mayor porcentaje de alumnos $(85,2 \%)$ consideró como violenta. Sin embargo, es una de las situaciones que, según ellos, sucede con menor frecuencia. Solo un $2,5 \%$ de los estudiantes sostiene que esta situación se da siempre o casi siempre en sus escuelas (García, 2009). La tenencia de armas en la escuela es la situación tipificada como violenta por el mayor número de estudiantes; sin embargo, es la que reconocen como menos habitual (Observatorio de Violencia en las Escuelas, 2009; García, 2009).

Los casos de agresión y muerte con armas en la escuela son excepcionales, pero conmueven nuestras representaciones sobre la escuela como un lugar pacificado. Observemos cómo fue representado en una nota del caso:

"La repercusión en España. El diario español El Mundo tituló en su portal de Internet: 'Robó el arma a su padre, agente de seguridad. Un estudiante de 15 años mató a tiros a tres compañeros de escuela en una ciudad argentina'. El ABC de Madrid dijo: 'Estudiante mató a tres compañeros e hirió 
Revista de la Escuela de Ciencias de la Educación, año 13, nRo. 12, vol. 2, Julio a diciembre de 2017. PÁginas 13-33. ISSN 1851-6297. ISSN 2362-3349 (EN LINNEA). LA SENSIBILIDAD POR LAS VIOLENCIAS EN EL ESPACIO ESCOLAR DESDE EL DISCURSo mediático. ViRginia Saez y Verónica Soledad Silva

a cinco en un tiroteo'. El caso en la prensa italiana. El diario Corriere Della Sera sostuvo: 'Argentina, alumno dispara en escuela: tres muertos'. En la crónica afirma que el joven 'sembró el terror' y despliega un antecedente: compara 'las escenas de terror' con la tragedia ocurrida en abril de 1999 en una escuela secundaria de Columbia". (Extra, 29-09-2004).

Posteriormente, las prácticas discursivas de la prensa platense visibilizan la presencia de armas en el espacio escolar como una forma diferenciada de violencia entre compañeros.

Como se destacó anteriormente, diversas investigaciones sobre el fenómeno a nivel nacional (Kaplan, 2006; Observatorio de Violencia en las Escuelas, 2009) sostienen que las violencias en la escuela no se caracterizan habitualmente por el uso de armas ni por hechos graves de este tipo. Lo más frecuente son las prácticas discriminatorias, los hechos de maltrato entre compañeros (las burlas, el ser ignorado, la ridiculización, el hablar mal del otro, el mirarse mal, etc).

Es decir que en las instituciones escolares son excepcionales las situaciones tipificadas por el código penal, pero el discurso de los diarios promueve una mirada totalizante sobre el hecho, promoviendo sensibilidades vinculadas, a la impotencia, el miedo y un temor generalizado, deslizando sentidos acerca de si esto pasó en la escuela entonces todo es posible.

Señalamos que aún en casos de un calibre grave como este, es necesario considerarlos bajo el marco de los derechos de los niños, niñas y adolescentes. Es necesario que los medios de comunicación hagan referencia a la condición ciudadana de las y los jóvenes y a los derechos inalienables que les corresponden en tal sentido.

Otra estrategia vinculada con la anterior es la presentación de diversos casos similares, generando una representación de "contagio". Veamos algunos ejemplos:

"A CASI SIETE MESES DE LA MASACRE Otro chico armado en Carmen de Patagones. Asomó el fantasma de Junior. Fue el lunes pasado. Un nene de 12 años tomó el revólver de su tío, se lo calzó en la cintura y fue al club de básquet. Después habría entrado a su escuela para exhibir el arma en un recreo. Las autoridades del colegio lo desmintieron, pero los padres de algunos alumnos radicaron la denuncia". (Hoy, 21-04-2005).

"Un alumno de séptimo grado del colegio Don Bosco de San Carlos de Bariloche, de 12 años, fue separado del establecimiento y puesto bajo tratamiento psicopedagógico, por concurrir a la escuela con una pistola calibre 22 y amenazar con el arma a sus compañeros". (El Día, 01-10-2004).

"En la provincia de Chubut, un joven de 17 años salía de clases en el turno tarde y comenzó a disparar con un arma en la puerta de una escuela del 
Revista de la Escuela de Ciencias de la Educación, año 13, nRo. 12, vol. 2, julio a diciembre de 2017. Páginas 13-33. ISSN 1851-6297. ISSN 2362-3349 (EN LINEA). LA SENSIBILIDAD POR LAS VIOLENCIAS EN EL ESPACIO ESCOLAR DESDE EL DIScurso mediático. Virginia Saez y Verónica Soledad Silva

Corradi en la ciudad de Puerto Madryn. Fue detenido por la Policía antes de que hubiera heridos. En la provincia de San Juan, en la localidad de Rivadavia, un adolescente de 16 años exhibió un arma y amenazó a otro compañero. Ya fue apartado del colegio. Las autoridades del establecimiento explicaron que era un joven muy callado, y no era muy buen alumno". (Extra, 01-10-2004).

Lo que los medios dan a conocer no transita por un registro único, sino por varios, profundamente diferentes. En el caso de la Masacre de Carmen de Patagones aparecen modos de designar que apelan intencionalmente a la emotividad y los afectos.

Podemos observar de qué modos las representaciones de los discursos mediáticos aluden predominantemente al ámbito de las emociones. Como sostiene Rotker (2000), "es como si el vacío del lenguaje de la razón y el deterioro de los significantes buscara anclaje en el lenguaje de la subjetividad, de los sentimientos, lo que termina aumentando la difusa paranoia cotidiana" (p. 9).

Las emociones parecen ser personales a primera vista, pero, aunque no son determinados simplemente por la historia o la cultura, ellos son los resultados de legados complejos que vienen de transacciones complejas del pasado (Depelteau, 2008). Así cómo en las notas anteriores pudimos observar cómo la interpelación afectiva estaba puesta en el lugar del miedo y el temor, en los siguientes ejemplos observamos cómo se busca generar identificación con el dolor de las víctimas, sus familiares y la comunidad en general.

"Dolor: el pueblo entero despidió los restos de los tres chicos asesinados". (Hoy, 30-09-2004).

“EL ÚLTIMO ADIÓS. (...) El cortejo fúnebre fue encabezado por el intendente de la ciudad y centenares de vecinos. En la emotiva ceremonia también participó uno de los chicos que sufrió heridas. Otros veinticinco alumnos de la misma división no pudieron llegar al cementerio, debido a que sufrieron una descompensación". (Hoy, 30-09-2004).

"Al ser consultada acerca de la masacre, Saldías opinó que Rafael podría haber 'explotado de otra manera', aunque enseguida aclaró: 'no es un victimario, sino una víctima'. '¿Cómo vivirá cuando tomé conciencia de todo lo que pasó, o lo que están pasando su mamá y su papá?', se preguntó Saldías. 'No sé cómo sucedió (el ataque del martes), pero no juzgamos a nadie, ni vamos a agredir al nene, porque no se puede hablar a la ligera de todo esto"'. (El Día, 02-10-2004).

Hay un énfasis en la espectacularidad de los relatos, y sus modos discursivos y comunicacionales apelan a la emotividad instantánea, dejando a veces 
Revista de la Escuela de Ciencias de la Educación, año 13, nRo. 12, vol. 2, Julio a diciembre de 2017. PÁginas 13-33. ISSN 1851-6297. ISSN 2362-3349 (EN LINNEA). LA SENSIBILIDAD POR LAS VIOLENCIAS EN EL ESPACIO ESCOLAR DESDE EL DISCURSo mediático. ViRginia Saez y Verónica Soledad Silva

poco margen a la reflexividad (Brener, 2009). Así, se presentan adjetivaciones como:

"Parece increíble pensar que la ira de un jovencito de apenas 15 años le haya costado la vida a tres de sus compañeritos. Es inconcebible también creer que más de veinte jovencitos hayan conseguido escapar ilesos por una puerta de apenas 70 centímetros de ancho". (Hoy, 30-09-2004).

Tal como se desprende de los extractos analizados, los medios de comunicación juegan un papel destacado en la configuración de los ejes sobre los que se articulan las figuras de lo amenazante y los sentimientos que ellas despiertan; y por ende, tienen una importante responsabilidad.

\section{Consideraciones finales}

El discurso mediático es un registro y un lugar de lucha simbólica en la construcción de la sensibilidad por las violencias en el espacio escolar. Haciendo foco en el contexto argentino tomamos el caso Carmen de Patagones en tanto constituye un hito en la configuración del campo representacional en la prensa platense.

En la singularidad de este acontecimiento emergen ciertas enunciaciones y visibilidades. La descripción y análisis de su surgimiento implica una nominalización. Desde una mirada individualizante se enuncian posibles soluciones para abordar esta temática, focalizando en la figura del victimario, su estructura psicológica y el posible destino del joven (la cárcel o el manicomio).

El análisis socioeducativo se centró en cómo las narrativas que construyen los medios se fundamentan principalmente en una racionalidad biomédica legitimando nuevos sentidos sobre la escuela y los sujetos que la habitan. En ellas las experiencias de violencia en la escuela son del orden de lo individual, ya sea biológico o psicológico. Esto nos remite nuevamente a pensar en la relación individuo-sociedad. Como se abordó anteriormente la individualidad está determinada por la estructura de la sociedad en la que se nace, por lo cual los comportamientos individuales necesitan ser abordados en una matriz social (Elias, 2009). Los comportamientos violentos y las prácticas de pacificación se anclan en procesos civilizatorios de sociedades particulares con individuos concretos (Castorina y Kaplan, 2006).

Estas visiones de las trayectorias sociales y educativas esencializan los episodios violentos en el espacio escolar en determinados sujetos. Una mirada dialéctica sobre el par individuo-sociedad permitirá redireccionar críticamente esta cuestión.

Tras el proceso analítico observamos la presencia de las voces autorizadas de funcionarios e intelectuales externos a la escuela para analizar el fenómeno. Desde voces reconocidas se definen prácticas hegemónicas sobre la escuela y los actores que la habitan. Así, las causas del fenómeno de las 
Revista de la Escuela de Ciencias de la Educación, año 13, nRo. 12, vol. 2, JUlio a diciembre de 2017. Páginas 13-33. ISSN 1851-6297. ISSN 2362-3349 (EN LINEA). LA SENSIBILIDAD POR LAS VIOLENCIAS EN EL ESPACIO ESCOLAR DESDE EL DIScurso mediático. Virginia Saez y Verónica Soledad Silva

violencias suelen situarse en un individuo que aparece prácticamente deshistorizado y descontextualizado. Por tal motivo es relevante su análisis para contribuir a la desustancialización de la violencia.

Desde un discurso individualizante (Kaplan, 2006), se propone una mirada de los hechos reductible al par víctima-victimario, y visibilizando soluciones del orden de lo singular a los episodios. Son actos de nombramiento y clasificación que operan distinguiendo individuos y grupos, y que cobran adhesión en el pensamiento social y en la producción de subjetividad social. Esta forma de describir el fenómeno es interceptada con un discurso emotivo, donde se presenta a las víctimas como una comunidad de sufrientes (Goffman, 2001) y a los victimarios como causantes de ese dolor.

La forma de abordar este acontecimiento implica un posicionamiento en ese momento del pasado que conforma el presente. La Masacre de Carmen de Patagones en tanto acontecimiento (Foucault, 2004) muestra como se configuran históricamente los discursos sobre las violencias en el espacio escolar. Produce un cambio en el campo representacional del espacio escolar en los medios de comunicación. El presente estudio es de relevancia para documentar y analizar la construcción discursiva, poner en cuestión y entretejer el sentido de los hechos. Dado que hay violencias con un alto nivel de naturalización que no se perciben como tales (violencia de la pobreza, de los niños abusados), y no tienen registro en el discurso mediático. En la prensa platense, desde el acontecimiento de la Masacre de Carmen de Patagones se produjeron cambios en la sensibilidad por las violencias en el espacio escolar, específicamente desde este caso, se visibiliza la presencia de armas en el espacio escolar como una forma diferenciada de violencia entre compañeros. $Y$ se invisibilizan otras formas, como las prácticas discriminatorias. Por lo tanto, este análisis constituye una contribución en el análisis de las sensibilidades por la violencia en determinado tiempo histórico y social.

Constituye un aporte especifico al análisis de los modos en que la prensa escrita nos coloca frente a datos y episodios que refuerzan una serie de creencias sociales que configuran un sentido práctico de la doxa punitiva (Kaplan, 2006). Reconstruir estos procedimientos permite desandar el camino de la naturalización, característico de la doxa (Bourdieu y Eagleton, 2000), para dar cuenta críticamente de algunos de los mecanismos más importantes de construcción social del sentimiento de inseguridad en las escuelas a partir de los hechos de violencia y de sus consecuencias en el tratamiento de la cuestión del alumno violento. Estos argumentos conforman un insumo valioso en la alfabetización mediática en la formación docente.

Es importante que los medios de comunicación participen en el desarrollo de nuevas sensibilidades y disposiciones hacia los jóvenes como sujetos de derecho y hacia las escuelas como instituciones formadoras, contribuyendo a renovar el compromiso social con una sociedad más democrática y más justa. 
Desde una mirada sociohistórica, sostenemos que es necesario considerar que los medios de comunicación construyen representaciones sobre la realidad y operan en las formas sentir y habitar el mundo.

A modo de cierre es importante destacar la relevancia de esta investigación en tanto es el primer estudio en Argentina que aborda las representaciones mediáticas del caso de Carmen de Patagones desde una perspectiva socioeducativa y tomando como insumo la prensa local de la Ciudad de La Plata. En tal sentido, amplía la base empírica con la que pensar y discutir cómo a través de ciertos hitos en las coberturas se colabora en la construcción de miradas estigmatizantes sobre los sujetos escolares.

\section{Notas bibliográficas}

(1) La investigación de Saez (2016) constituye una tesis de doctorado que caracterizó y analizó, desde una perspectiva socioeducativa, las prácticas discursivas producidas por la prensa escrita de la ciudad de La Plata sobre el fenómeno de las violencias en la escuela, en el período 1993-2011.

(2) En este trabajo usaremos el término "violencias" en plural (Kaplan, 2006) con el objeto de dar cuenta de la multiplicidad de fenómenos que atraviesan la trama escolar.

(3) Principalmente los desarrollos plasmados en una de sus obras más importantes: El proceso civilizatorio. Investigaciones Psicogenéticas y sociogenéticas.

(4) Su investigación analiza las transformaciones del comportamiento de las clases altas de Europa occidental: los guerreros nobles y la nobleza cortesana, estableciendo un recorte temporal desde la baja Edad Media y Renacimiento hasta la Edad Moderna. Elias (2009) señala que a partir de la baja Edad Media se desarrolla un aumento paulatino del autocontrol individual.

(5) El 7 de octubre de 2004, el Gobierno Nacional, junto con la Unesco y la Universidad Nacional de San Martín crearon el Observatorio Argentino de Violencia en las Escuelas.

\section{Referencias bibliográficas}

- Alba, G. (2002). Los niños en la prensa colombiana del crimen. Signo y Pensamiento, 21 (41), pp. 107-119.

- Baczko, B. (1991). Los imaginarios sociales. Buenos Aires: Nueva Visión.

- Bleichmar, S. (2008).Violencia social - Violencia escolar. De la puesta de límites a la construcción de legalidades. Buenos Aires: Noveduc.

- Bonilla Vélez, J. I. y Tamayo Gómez, C. A. (2007). Las violencias en los medios, los medios en las violencias, núm. 1, Bogotá: CINEP, Fundación Centro de Investigación y Educación Popular.

- Bourdieu, P. (1997). Entrevista a Pierre Bourdieu, realizada por P. R. Pires y publicada en O Globo (Río de Janeiro) el 4 de octubre de 1997. Traducción: F. Sanabria y G. Vargas.

- Bourdieu, P. (1988a). Espacio social y poder simbólico. En Cosas dichas. Buenos Aires: Gedisa.

- Bourdieu, P. (1998b). Sociología y democracia. En Capital cultural, escuela y espa- 
Revista de la Escuela de Ciencias de la Educación, año 13, nRo. 12, vol. 2, julio a diciembre de 2017. Páginas 13-33. ISSN 1851-6297. ISSN 2362-3349 (EN LINEA). LA SENSIBILIDAD POR LAS VIOLENCIAS EN EL ESPACIO ESCOLAR DESDE EL DIS-

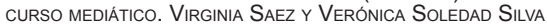

cio escolar. (Pp. 46-51) Madrid: Siglo XXI.

- Bourdieu, P. (2001). ¿Qué significa hablar? Economía de los intercambios lingüísticos. Barcelona: Akal.

- Bourdieu, P. (2002). Cuestión de Palabras. Una visión más modesta del rol de los periodistas. Intervención en coloquio de Periodistas sin fronteras, publicada en (1992). Les mensonges du Golfe [Las mentiras del Golfo]. París: Arlèa. Esta conferencia se encuentra traducida en la recopilación Bourdieu, P. (2005). Pensamiento y acción. Buenos Aires: Libros del Zorzal.

- Bourdieu, P. (2006). Intelectuales, política y poder. Buenos Aires: Eudeba.

- Bourdieu, P. y Eagleton, T. (2000). Doxa y vida ordinaria. Pensamiento crítico contra la dominación, New Left Review, 0 (1), enero, pp. 219-231.

- Bourdieu, P. y Wacquant, L. (2005). El propósito de la sociología reflexiva. En Una invitación a la sociología reflexiva. Buenos Aires: Siglo XXI.

- Brener, G. (2009). Violencia y escuela como espectáculo. La relación medios-sociedad. En Carina V. Kaplan (Dir.), Violencia escolar bajo sospecha. (Pp. 199-240). Buenos Aires: Miño y Dávila.

- Castorina, J. A. y Kaplan, C. (2006). Violencias en la escuela: una reconstrucción crítica del concepto. En Kaplan, C. (Dir.), Violencias en plural. Sociología de las violencias en la escuela. (Pp. 27-54).Buenos Aires: Miño y Dávila.

- Comité Federal de Radiodifusión (Comfer) (2005). Índice de violencia de la televisión argentina. Buenos Aires. Consultado el 19 de diciembre de 2014 en la<www.comfer. gov.ar>.

- Consejo Nacional de Televisión de Chile (1998). Cinco estudios sobre violencia y televisión en Chile. Santiago de Chile: Consejo Nacional de Televisión de Chile.

- Consejo Nacional de Televisión de Chile (2002). Barómetro de violencia $N^{\circ}$ 2: Noticieros 2002. Santiago de Chile: Consejo Nacional de Televisión de Chile.

- Delgado, F. J. (1998). Comunicación, inseguridad ciudadana y control social. Comunicación. Estudios Venezolanos de Comunicación, 103 (1), agosto.

- Depelteau, F. (2008). Hacia una teoría crítica y relacional: de la emancipación egocéntrica a la relacional. En Carina V. Kaplan (coord.), La civilización en cuestión. Escritos inspirados en la obra de Norbert Elias. (Pp. 187-236) Buenos Aires: Miño y Dávila.

- Elias, N. (2009). El proceso de la civilización. México: Fondo de Cultura Económica.

- Ford, A. (1999). La marca de la bestia. Identificación, desigualdades e infoentretenimiento en la sociedad contemporánea. Buenos Aires: Norma.

- Foucault, M. (1983). Debates con los historiadores. En Michael Foucault. El discurso del poder. (pp. 216-232). Buenos Aires: Folios ediciones.

- Foucault, M. (1992). Nietzsche, la genealogía, la historia. En Microfísica del poder. Madrid: Ediciones la piqueta.

- Foucault, M. (2004). La arqueología del saber. Buenos Aires: Siglo XXI.

- Garriga Zucal, J. y Noel (2010). Notas para una definición antropológica de la violencia: un debate en curso. PUBLICAR-En Antropología y Ciencias Sociales, 0(9), 97-121.

- García, S. (2009). La experiencia emocional de los estudiantes secundarios. En Ca- 
rina V. Kaplan (Dir.), Violencia escolar bajo sospecha. (pp. 81-136). Buenos Aires: Miño y Dávila.

- Giroux, H. A. (1997). Cruzando límites: Trabajadores culturales y políticas educativas. España: Paidós.

- Goffman, E. (2001). Estigma la identidad deteriorada. Buenos Aires: Amorrortu.

- González Bernaldo de Quirós, P. (2008). La "sociabilidad" y la historia política, Nuevo Mundo Mundos Nuevos [En linea], Bibliothèque des Auteurs du Centre. Disponible en: http://nuevomundo.revues.org/24082

- Herrera, A. (1998). Influencia de la guerra civil en El Salvador en el desarrollo de la prensa nacional (1980-1992). En Revista Latina de Comunicación Social, $\mathrm{n}^{\circ} 1$.

- Iglesias, A. (2015). ¿Todo tiempo pasado fue mejor? Un análisis de la formación y el desempeño de los nuevos docentes y sus representaciones en la prensa gráfica argentina (2000-2013). Revista mexicana de investigación educativa, 20(64), 123-151.

- Kaplan, C. (Dir.) (2006). Violencias en plural. Sociología de las violencias en la escuela. Buenos Aires: Miño y Dávila.

- Kaplan, C. (Dir.) (2009). Violencia escolar bajo sospecha. Buenos Aires: Miño y Dávila.

- Kaplan, C. (2011). Sobre la tolerancia, los umbrales y los miedos: Las transformaciones en la sensibilidad sobre la violencia en la educación escolar. En F. Hillert (comp.). La mirada pedagógica para el siglo XXI: teorías, temas y prácticas en cuestión: reflexiones de un encuentro. (pp. 75-84). Buenos Aires: Editorial de la Facultad de Filosofía y Letras Universidad de Buenos Aires.

- Kaplan, C. (2013). Imagens sobre mobilizações juvenis e estudantis: a criminalização através dos meios de comunicação. Revista de Estudos Universitários (REU), 39 (2), diciembre, 293-309.

- Kaplan, C. y Silva V. (2016). Respeto y procesos civilizatorios. Imbricación socio-psíquica de las emociones. Praxis educativa, 20 (1), 28-36.

- Lavena, C. (2002). ¿La violencia va a la escuela? Una mirada a la violencia escolar en la Argentina. Tesis de Maestría en Educación, Universidad de San Andrés, Argentina.

- López, R. y Cerda, A. (2001). Violencia en la televisión mexicana: un análisis del contenido de los treinta programas con mayor audiencia. Hiper-textos, 2 (1).

- Loreti, D. (2003). Los desafíos del derecho de la información como herramienta de la política del Estado hacia la comunicación. Zigurat, 4 (4), noviembre, 33-42.

- Martín Criado, E. (2014). Mentiras, inconsistencias y ambivalencias. Teoría de la acción y análisis de discurso. En Revista Internacional de Sociología, 72 (1), eneromarzo, 115-138.

- Martini, S. (2004). Periodismo, noticia y noticiabilidad. Buenos Aires: Norma.

- Míguez, D. y Noel, G. (2006). Entre el pánico moral y el suceso real: la violencia escolar en la Argentina reciente. Ponencia presentada en el VIII Congreso Argentino de Antropología Social, Salta, septiembre.

- Núñez, T. (1999). Los profesores vistos por la prensa: de la realidad al mito social. Comunicar, 12 (1), 47-54.

- Observatorio Argentino de Violencia en las Escuelas (2009). Violencia en las es- 
Revista de la Escuela de Ciencias de la Educación, año 13, nRo. 12, vol. 2, julio a diciembre de 2017. Páginas 13-33. ISSN 1851-6297. ISSN 2362-3349 (EN LINEA). LA SENSIBILIDAD POR LAS VIOLENCIAS EN EL ESPACIO ESCOLAR DESDE EL DIS-

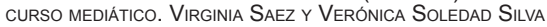

cuelas. Investigaciones, resultados y políticas de abordaje en prevención. Simposio Francia - Argentina. Consultado el 19 de diciembre de 2014 en la<http://ceapi.files. wordpress.com/2012/04/violencia2.pdf>.

- Rotker, S. (2000).Ciudadanías del miedo. Caracas: Nueva Sociedad.

- Saintout, F. (2009). ¿Culturas violentas? La producción mediática de violencias legítimas/ilegítimas y de sujetos viables/inviables. El caso de las juventudes. Consultado el 19 de diciembre de 2014 en lawww.perio.unlp.edu.ar/observatoriodejovenes/archivos/culturasviolentas.pdf.

- Sabino, C. (1996). El proceso de investigación. Buenos Aires: Lumen.

- Saez, V. (2016). Prácticas discursivas de la mediatización de la violencia en espacios escolares. Tesis de Doctorado en Educación, no publicada, Facultad de Filosofía y Letras, Universidad de Buenos Aires, Argentina

- Saez, V. (2015). Una mirada a la investigación sobre medios, violencia y escuela. Revista Entramado, 11 (1), 136-155.

- Unicef y FLaCSo (2011). Clima, conflicto y violencia en las escuelas. Argentina: FLaCSo. Consultado el 19 de diciembre de 2014 en la<www.unicef.org/argentina/ spanish/clima_conflicto_violencia_escuelas.pdf>.

- Vasilachis de Gialdino, I. (2004). El lenguaje de la violencia en los medios de comunicación. Las otras formas de ser de la violencia y la prensa escrita. Argentina, Consejo Nacional de Investigaciones Científicas y Técnicas (Conicet). Consultado el 19 de diciembre de 2014 en la<http://biblioteca.secyt.gov.ar>.

- Verón, E. (1987). Prefacio a la segunda edición. En Construir el acontecimiento. Buenos Aires: Gedisa.

- Verón, E. (1981). La presse. Produit, production, reception, núm. 1, París: Didier Erudition.

\section{Fuentes}

- www.eldia.com.ar

- www.diariohoy.net

- www.diagonales.com

- www.diarioextra.com.ar 